\title{
An Insight into the Characteristic Equation for an Integer Program
}

\author{
Santosh Kumar \\ Department of Mathematical and Geospatial Sciences, \\ School of Sciences, RMIT University, Melbourne, Australia. \\ and \\ Department of Mathematics and Statistics, \\ University of Melbourne, Australia. \\ Corresponding author: santosh.kumarau@gmail.com \\ Elias Munapo \\ School of Economics and Decision Sciences, \\ North West University, Mafikeng Campus, Mafikeng, South Africa. \\ E-mail: emunapo@gmail.com \\ Philimon Nyamugure \\ Department of Statistics and Operations Research, \\ National University of Science and Technology, \\ PO Box AC 939, Ascot, Bulawayo, Zimbabwe. \\ E-mail: philimon.nyamugure@ nust.ac.zw \\ (Received July 15, 2020; Accepted September 20, 2020)
}

\begin{abstract}
This article enhances properties and applications associated with the characteristic equation (CE) developed to find an optimal and other ranked-optimal solutions of linear integer programming model. These enhanced properties have applications in the analysis of the multi-objective linear integer programs. The paper also identifies why the CE approach is not possible for some special linear programming (LP) models and creates a challenge for further investigation.
\end{abstract}

Keywords- Characteristic equation, Ranked-optimal solutions, Integer polyhedron.

\section{Introduction}

In the context of a pure integer programming model, Kumar et al. (2007) developed a conceptual idea for determination of an integer optimal solution to an integer program. They first solved the given integer linear programming (ILP) as a LP and utilised the final output of the relaxed linear program and developed a new linear relation to find an optimal solution to that pure integer programming problem. Unlike the cutting plane approaches, for example, see (Gomory, 1958) and its variants (Schrijiver, 1986). Kumar et al. (2007) developed a linear hyperplane form the simplex output and reached directly to the optimal integer solution for the given linear integer program. In that study this linear relation was called by them "A descending hyperplane", as that hyperplane not only provided them an optimal integer solution to the given linear integer program, but their approach was also able to find ranked-optimal solutions in a descending order. In other words, they obtained the optimal solution, and if required they were also able to identify the ranked optimal solutions, i.e. the $2^{\text {nd }}, 3^{\text {rd }}$ best solutions, etc. In a subsequent paper, the linear descending hyperplane was renamed by Kumar and Munapo (2012) as a characteristic equation (CE) for integer optimal solutions. They also pointed out that the CE is a necessary but not a sufficient condition for existence of an integer optimal solution. The special feature of $\mathrm{CE}$ was that it was able to provide 
International Journal of Mathematical, Engineering and Management Sciences

Vol. 6, No. 2, 611-620, 2021

https://doi.org/10.33889/IJMEMS.2021.6.2.037

not only the optimal solution but was also able to provide $k^{\text {th }}$ best optimal solutions, ( $k \geq$ $2, i . e ., k=2,3, \ldots)$. This feature has not been seen in other optimization techniques. These ordered optimal solutions play a useful role to find non-dominated point set for a bi-objective and multi-objective linear integer program. Using the CE approach, Al-Rabeeah et al. (2019) obtained a non-dominated point set for a bi-objective linear integer problem, and Al-Hasani et al. (2020) developed a rank-based solution method for finding a non-dominated point set of a given multiobjective integer program. Nyamugure et al. (2017) applied the CE concept for a mixed integer program and Kumar et al. (2009) solved a binary integer program using the CE approach.

This paper attempts to provide further insight in the development and applications of the CE for a pure integer program.

In this paper, the authors have obtained a few more interesting results, which are discussed in section 3. These properties have applications in multi-objective linear integer programs, see AlHasanai et al. (2020). The paper has been organized in 5 sections. For completeness of the paper, a brief derivation of the characteristic equation has been presented in section 2 . In section 4 , we have presented a few numerical illustrations to demonstrate each feature discussed in section 3. Finally, the paper has been concluded in section 5 .

\section{Brief Review of the Characteristic Equation}

Consider a given linear integer program:

$\operatorname{Max} z=\sum_{j=1}^{n} c_{j} x_{j}$

Subject to: $\sum_{j=1}^{n} a_{i j} x_{j} \leq b_{i}, i=1,2, \ldots, m$ and

$x_{j} \geq 0$ and integer for all $j$.

The LP relaxation of the model (1), i.e. removing integer requirement on all variables, can be solved by using any LP software. Consider the objective function row of the final simplex tableau gives:

$z+a_{m+1,1} s_{1}+a_{m+1,2} s_{2}+\cdots+a_{m+1, n} s_{n}=P$

Here $s_{1}, s_{2}, \ldots, s_{n}$ are non-basic variables and $a_{m+1,1}, a_{m+1,2}, \ldots, a_{m+1, n}$ are the non-negative coefficients of the non-basic variables in the objective function row and $P$ is the value of the objective function $z$ under the condition that all non-basic variables are zero at the optimal solution. The characteristic equation was developed from the LP output (2), i.e. from the objective row in the final simplex tableau. These coefficients in the final simplex table are non-negative quantities i.e. $a_{m+1,1}, a_{m+1,2}, \ldots, a_{m+1, n} \geq 0$ but they can be integer, zero or fractional values, hence one can re-write equation (2) in the form of equation (3):

$\left(\frac{D}{D}\right) z+\frac{\alpha_{1} s_{1}+\alpha_{2} s_{2}+\cdots+\alpha_{n} s_{n}}{D}=\frac{P^{\prime}}{D}=\frac{R+i D}{D}$

Note that $a_{m+1, j}=\frac{\alpha_{j}}{D}, j=1,2, \ldots, n, D>0$ is the common denominator, $R$ is the integer residue of $\left(\frac{P^{\prime}}{D}\right)$ and $\alpha_{j}$ represents integer coefficients for the non-basic variables $s_{j}, j=1,2, \ldots, n$.

From equation (3), Kumar et al. (2007) obtained the CE given in equation (4) 
International Journal of Mathematical, Engineering and Management Sciences

Vol. 6, No. 2, 611-620, 2021

https://doi.org/10.33889/IJMEMS.2021.6.2.037

$\sum_{j=1}^{n} \alpha_{j} s_{j}=R+i D, i=0,1,2, \ldots,\left\lceil\frac{p^{\prime}}{D}\right\rceil$

The CE in equation (4) is used to search for an integer optimal solution and other ranked-optimal solutions to given integer program.

\section{Further Considerations into Development and Applications of the $\mathbf{C E}$}

A CE can be developed after each simplex iteration, which can identify the integer points within the integer polyhedron developed by the LP constraints of the given LIP model (1).

At the end of each simplex iteration, one obtains an objective row, a relation, which is like the relation (2). It is given by:

$z+A_{m+1,1} s_{1}+A_{m+1,2} s_{2}+\cdots+A_{m+1, n} s_{n}=O b_{\text {value }}$

Here the coefficients $A_{m+1, j}$ in equation (5) may be zero, positive or negative quantities. These values become non-negative in the final simplex table when the LP solution has attained its optimum value. Once again, one can re-write from equation (5) with a common denominator as was done in (3) and obtain a new relation like equation (4) given below as equation (6):

$\sum_{j=1}^{n} \beta_{j} s_{j}=r+i d, i=0,1,2, \ldots,\left\lceil\frac{p *^{\prime}}{d}\right\rceil$

Note that all elements in (6) are integer quantities but these $\beta_{j}$ 's are not restricted to non-negative values; they can be positive, zero or negative integers. Equation (6) holds properties like the CE. It can also identify integer points in the convex polyhedron. This is illustrated in Section 4, Example 1.

\subsection{Integer Polyhedron and Feasible Integer Points are Independent of the Objective Function}

The optimal integer solution is an extreme point of the integer polyhedron, where the objective function attains its maximum value. All other integer points in the feasible space belong to the class of ranked-optimal solutions. The CE can identify these ranked-optimal solutions. However, if the objective function is modified, ranks with respect to the given objective function may not be maintained but feasibility of all integer points will remain unchanged. This fact may be useful in situations, discussed below

$$
\text { A CE with } R=0, D=1 \text { and } \alpha_{j}=1 \text { for one or more } j
$$

In a LP solution, if all elements in the objective row are integer values, the $\mathrm{CE}$ will have residue ' $R=0$ ' and the common denominator $D=1$. In addition, if any $\alpha_{j}=1$, the number of potential solutions to that $\mathrm{CE}$ increases rapidly, resulting in increased computational load. In such circumstances, it may be desirable to carry out the search by using a modified objective function and, once feasible integer points have been identified, they can be evaluated again with respect to the given objective function and re-arranged in a descending order.

For the above situation, consider that the objective function row in the final simplex tableau is free of fractional values. Consequently, we will have $D=1, R=0$ and $\alpha_{j}=a_{m+1, j}, j=1,2, \ldots, n$. Therefore, the CE equation (4) will become: 
International Journal of Mathematical, Engineering and Management Sciences

Vol. 6, No. 2, 611-620, 2021

https://doi.org/10.33889/IJMEMS.2021.6.2.037

$\sum_{j=1}^{n} \alpha_{j} s_{j}=i, i=0,1,2, \ldots,\left\lceil\frac{p^{\prime}}{D}\right\rceil$

It may be noted that the search process for the $\mathrm{CE}$ (7) will have to be carried out for each value of $i=0,1,2$, . This search will become time consuming if $\alpha_{j}=a_{m+1, j}=$ 1 for anyone j or many $j^{\prime} s$. However, this performance, in some cases may be enhanced by a minor modification in the values of the coefficients of basic variables in the given objective function. If we change these coefficients within sensitivity limits, optimal solution point will not change but descending order of ranked solutions may change. This aspect is illustrated in Section 4, Example 2.

\subsection{Integer Point Search for a Multi-objective Linear Integer Program}

In a bi-objective linear integer program, one central problem is to identify the non-dominated point set. When the CE is in the form of equation (7), search efficiency for feasible integer points can be enhanced by using a modified objective function as feasible integer points are independent of the objective function. Consider two objective functions for a bi-objective integer program denoted by $z_{1}$ and $z_{2}$. Further assume that we have identified some of the ranked-optimal integer solutions with respect to the objective $z_{1}$, i.e. $k^{\text {th }}$ ranked - optimal solution denoted by $z_{1}^{1} \geq z_{1}^{2} \geq \cdots \geq$ $z_{1}^{k}$. The LP convex space defined by $A X \leq b, X \geq 0$ can be seen as a union of three separate convex subregions defined by:

Region 1: $A X \leq b, z \geq z_{1}^{1}$ (This region is free of integer points, assuming LP optimal solution is a fractional answer.)

Region 2: $A X \leq b, z_{1}^{k} \leq z \leq z_{1}^{1}$ (This is the region which has been scanned for the integer points with respect to the objective $z_{1}$.

Region 3: $A X \leq b, z \leq z_{1}^{k}-1$ (Since the last identified integer point corresponds to $z_{1}^{k}$, the unscanned region remaining is,$z \leq\left(z_{1}^{k}-1\right)$.

It may be noted that since the Region 1 has no integer points, hence no need to search for integer points with respect to the objective $z_{2}$ and the integer points in the Region 2, can again be used with respect to the objective $z_{2}$ as integer points are independent of the objective function. However, these points may not be in a descending order with respect to the objective $z_{2}$ but when re-evaluated with respect to the objective $z_{2}$, these can be rearranged in the descending order. Therefore, new search with respect to the objective $z_{2}$ will be confined only to the Region 3 , which is a sub-space of the given convex space. Furthermore, suppose the value of $z_{2}$ at the known integer

points be denoted by $z_{2 @ z_{1}^{1}}, z_{2 @ z_{1}^{2}}, \ldots, z_{2 @ z_{1}^{k}}$. These values can be easily rearranged in descending order. This is illustrated in Section 4, Example 3.

\subsection{Why a CE does not exist for Special LPs like an Assignment and Transportation Models?}

The concept of $\mathrm{CE}$ is based on the fact, that by varying the value of the slack variables in a linear inequality, one can move constraints to various parallel positions and the CE helps to find those integer values of the slack variables to reach to the desired integer point directly. However, this possibility does not exist in the case of special models like an assignment and transportation. In the case of these two models, the slack variables are equal to zero for each feasible integer point, and therefore moving constraints by changing values of slack variables is out of question. Therefore, the ranked-optimal solution of this kind of model needs an alternative approach. The CE approach 
International Journal of Mathematical, Engineering and Management Sciences

Vol. 6, No. 2, 611-620, 2021

https://doi.org/10.33889/IJMEMS.2021.6.2.037

will be applicable to any other unimodular linear integer models, with $\mathrm{R}=0$ and $\mathrm{D}=1$. This question of finding ranked-optimal solutions for these special LP models will be reconsidered in a subsequent publication.

\section{Numerical Illustrations}

\subsection{Example 1}

Consider the following trivial Problem (8) shown in Table 1 Max $z=3 x_{1}+2 x_{2}$,

Subject to: $2 x_{1}+3 x_{2} \leq 11, x_{1} \leq 4, x_{2} \leq 3, x_{1}, x_{2} \geq 0$ and integer.

Table 1. Simplex presentation of the problem (8).

\begin{tabular}{|c|c|c|c|c|c|c|}
\hline & $x_{1}$ & $x_{2}$ & $s_{1}$ & $s_{2}$ & $S_{3}$ & RHS \\
\hline$s_{1}$ & 2 & 3 & 1 & 0 & 0 & 11 \\
\hline$S_{2}$ & $1^{*}$ & 0 & 0 & 1 & 0 & 4 \\
\hline$s_{3}$ & 0 & 1 & 0 & 0 & 1 & 3 \\
\hline$z_{j}-c_{j}$ & -3 & -2 & 0 & 0 & 0 & 0 \\
\hline
\end{tabular}

The next table after the pivot will be as given as given in Table 2 .

Table 2. Improved table after the pivot operation.

\begin{tabular}{|c|c|c|c|c|c|c|}
\hline & $x_{1}$ & $x_{2}$ & $s_{1}$ & $s_{2}$ & $s_{3}$ & RHS \\
\hline$s_{1}$ & 0 & $3^{*}$ & 1 & -2 & 0 & 3 \\
\hline$x_{1}$ & 1 & 0 & 0 & 1 & 0 & 4 \\
\hline$s_{3}$ & 0 & 1 & 0 & 0 & 1 & 3 \\
\hline$z_{j}-c_{j}$ & 0 & -2 & 0 & 3 & 0 & 12 \\
\hline
\end{tabular}

The final table, after the pivot, will be as given in Table 3 .

Table 3. Final output of problem (8).

\begin{tabular}{|c|c|c|c|c|c|c|}
\hline & $x_{1}$ & $x_{2}$ & $s_{1}$ & $s_{2}$ & $s_{3}$ & RHS \\
\hline$x_{2}$ & 0 & 1 & $1 / 3$ & $-2 / 3$ & 0 & 1 \\
\hline$x_{1}$ & 1 & 0 & 0 & 1 & 0 & 4 \\
\hline$s_{3}$ & 0 & 0 & $-1 / 3$ & $2 / 3$ & 1 & 2 \\
\hline$z_{j}-c_{j}$ & 0 & 0 & $2 / 3$ & $5 / 3$ & 0 & 14 \\
\hline
\end{tabular}

The CE from Table 2, which is not an optimal solution, is given by: $-2 x_{2}+3 s_{2}=i$, as $D=$ 1 and $R=0$

For analysis, consider $i=0$, results in $x_{2}=s_{2}=0$ giving the integer solution as in Table 2 .

For $i=1$, there is no solution and for $i=2$, the solution is given by:

$$
x_{2}=-1, \text { which is infeasible, hence abandened } \text {. }
$$

For $i=3$, the solution is given by $s_{2}=1, x_{2}=0, x_{1}=3, s_{1}=5, s_{3}=3$ and $z=9$.

The same integer point $\left(x_{1}, x_{2}\right)=(3,0)$ can again be reached from the CE obtained from Table 3 , 
International Journal of Mathematical, Engineering and Management Sciences

Vol. 6, No. 2, 611-620, 2021

https://doi.org/10.33889/IJMEMS.2021.6.2.037

which is given by $2 s_{1}+5 s_{2}=3 i$, as $D=3$ and $R=0$. Here are a few ranked optimal integer solutions obtained from Table 3 . The basic and non-basic have the relations, as given below:

$$
\begin{aligned}
& x_{2}+\left(\frac{1}{3}\right) s_{1}-\left(\frac{2}{3}\right) s_{2}=1 \\
& x_{1}+s_{2}=4 \\
& s_{3}-\left(\frac{1}{3}\right) s_{1}+\left(\frac{2}{3}\right) s_{2}=2
\end{aligned}
$$

Using the CE and the relations (9), (10) and (11), we get the following ordered-optimal solutions as given in Table 4. Note that the solution $(3,0)$, obtained earlier is contained in Table 4, as was expected.

Table 4. A few integer optimal solutions to problem (8).

\begin{tabular}{|c|c|c|c|c|}
\hline$i$ & RHS & Solution point & $z^{k}$ & Remarks \\
\hline 0 & $3 i$ & $x_{1}, x_{2}, s_{1}, s_{2}, s_{3}$ & Optimal \\
\hline 1 & 0 & $(4,1,0,0,2)$ & 14 & No solution \\
\hline 2 & 3 & & & $2^{\text {nd }}$ best \\
\hline 3 & 6 & $(4,0,3,0,3)$ & 12 & $3^{\text {rd }}$ best \\
\hline 4 & 9 & $(3,1,2,1,2)$ & 11 & $4^{\text {th }}$ best \\
\hline 5 & 12 & $(2,2,1,2,1)$ & 10 & $5^{\text {th }}$ best \\
\hline
\end{tabular}

\subsection{Example 2: The Objective Function can be Modified, if Desired.}

Consider a linear programming problem taken from Shogan (1988):

$\operatorname{Max} z=30 x_{1}-10 x_{2}+20 x_{3}-8 x_{4}$

Subject to:

$$
\begin{aligned}
& x_{1}-2 x_{2}+4 x_{3}+x_{4} \leq 7 \\
& -4 x_{1}+x_{2}-3 x_{3}+2 x_{4} \geq 3 \\
& -2 x_{1}-2 x_{3}+x_{4}=1 \\
& x_{1}+2 x_{2}+3 x_{3}+x_{4} \geq 12 \\
& x_{1}, x_{2}, x_{3}, x_{4} \geq 0 \text { and integer }
\end{aligned}
$$

The LP optimal solution to problem is given in Table 5. It may be noted that $x_{1}, x_{2}, x_{3}, x_{4}$ are given variables, $s_{1}$ is a non-negative slack variable, and $s_{2}, s_{4}$ are usual non-negative surplus variables with respect to (14) and (16).

The LP optimal solution from Table 5 is given by $x_{1}=17 / 6, x_{2}=5 / 4, x_{4}=\frac{20}{3}, s_{2}=\frac{1}{4}$ and the remaining non-basic variables $x_{3}=s_{1}=s_{4}=0$. The optimal value of the objective function is $115 / 6$. 
International Journal of Mathematical, Engineering and Management Sciences

Vol. 6, No. 2, 611-620, 2021

https://doi.org/10.33889/IJMEMS.2021.6.2.037

The CE and the relations between the basic and non-basic variables from Table 5 will be as follows:

$C E: 69 x_{3}+29 s_{1}+s_{4}=1+6 i, i=0,1, \ldots, 19$

Table 5. LP optimal solution of problem (12) to (17).

\begin{tabular}{|c|c|c|c|c|c|c|c|c|}
\hline BV & $x_{1}$ & $x_{2}$ & $x_{3}$ & $x_{4}$ & $s_{1}$ & $s_{2}$ & $s_{4}$ & RHS \\
\hline$x_{1}$ & 1 & 0 & $3 / 2$ & 0 & $1 / 6$ & 0 & $-1 / 6$ & $17 / 6$ \\
\hline$x_{2}$ & 0 & 1 & $-1 / 4$ & 0 & $-1 / 4$ & 0 & $-1 / 4$ & $5 / 4$ \\
\hline$x_{4}$ & 0 & 0 & 2 & 1 & $1 / 3$ & 0 & $-1 / 3$ & $20 / 3$ \\
\hline$s_{2}$ & 0 & 0 & $3 / 4$ & 0 & $-1 / 4$ & 1 & $-1 / 4$ & $1 / 4$ \\
\hline$z_{j}-c_{j}$ & 0 & 0 & $23 / 2$ & 0 & $29 / 6$ & 0 & $1 / 6$ & $115 / 6$ \\
\hline
\end{tabular}

The relations between the basic and non-basic variables from Table 5 will be given by:

$$
\begin{aligned}
& x_{1}+\left(\frac{3}{2}\right) x_{3}+\left(\frac{1}{6}\right) s_{1}-\left(\frac{1}{6}\right) s_{4}=\left(\frac{17}{6}\right) \\
& x_{2}-\left(\frac{1}{4}\right) x_{3}-\left(\frac{1}{4}\right) s_{1}-\left(\frac{1}{4}\right) s_{4}=\left(\frac{5}{4}\right) \\
& x_{4}+2 x_{3}+\left(\frac{1}{3}\right) s_{1}-\left(\frac{1}{3}\right) s_{4}=\left(\frac{20}{3}\right) \\
& s_{2}+\left(\frac{3}{4}\right) x_{3}-\left(\frac{1}{4}\right) s_{1}-\left(\frac{1}{4}\right) s_{4}=\left(\frac{1}{4}\right)
\end{aligned}
$$

From equation (18), for $i=0$, we get $69 x_{3}+29 s_{1}+s_{4}=1$, for which a feasible solution is $s_{4}=$ 1 , and $x_{3}=s_{1}=0$. This solution when substituted in relations (19) gives $x_{1}=3$, and next relation (20) results in a fractional answer $x_{2}=\left(\frac{6}{4}\right)=3 / 2$, hence search is abandoned and we move to next iteration.

For $i=1$, the CE relation will be: $69 x_{3}+29 s_{1}+s_{4}=7$.This gives $s_{4}=7$, which gives rise to $x_{1}=4, x_{2}=3, x_{4}=9, s_{2}=2, s_{4}=7, x_{3}=s_{1}=0, z=18$, the optimal integer solution.

\begin{tabular}{|c|c|c|c|c|}
\hline i value & CE RHS & CEsolution & Problem Solution & Remarks \\
\hline & & $x_{3}, s_{1}, s_{4}$ & $x_{1}, x_{2}, x_{4}, s_{2},(\mathrm{z})$ & \\
\hline 0 & 1 & $0,0,1$ & $3,3 / 2, \ldots$ & Fractional \\
\hline 1 & 7 & $0,0,7$ & $4,3,9,2(18)$ & Optimal \\
\hline 2 & 13 & $0,0,13$ & $5,9 / 4, .$. & Fractional \\
\hline 3 & 19 & $0,0,19$ & $6,6,13,5(16)$ & $2^{\text {nd }}$ Best \\
\hline 4 & 25 & $0,0,25$ & $7,30 / 4, \ldots$ & Fractional \\
\hline 5 & 31 & $0,1,2$ & $3,2,7,0(14)$ & $3^{\text {rd }}$ best \\
\hline 6 & 37 & $0,1,8$ & $4,14 / 4, \ldots$ & Fractional \\
\hline 7 & 43 & $0,1,14$ & $5,5,9,4(12)$ & $4^{\text {th }}$ best \\
\hline 8 & 49 & $0,1,20$ & $6,26 / 4$ & Fractional \\
\hline 9 & 55 & $0,1,26$ & $7,8,15,7(10)$ & $5^{\text {th }}$ best \\
\hline
\end{tabular}

A summary of ranked-optimal solutions obtained with the help of CE (18) for the problem defined by relations (12) to (17) is given in Table 6.

Table 6. Integer ranked-optimal solutions. 
International Journal of Mathematical, Engineering and Management Sciences

Vol. 6, No. 2, 611-620, 2021

https://doi.org/10.33889/IJMEMS.2021.6.2.037

Here we intend to illustrate that the CE (18) can be modified if $c_{1}$ value is altered from 30 to 26 (within the range of sensitivity analysis), the objective function row will change as shown in Table 7.

Table 7. Altered objective row.

\begin{tabular}{|c|c|c|c|c|c|c|c|c|}
\hline $\mathrm{BV}$ & $x_{1}$ & $x_{2}$ & $x_{3}$ & $x_{4}$ & $s_{1}$ & $s_{2}$ & $s_{4}$ & RHS \\
\hline$x_{1}$ & 1 & 0 & $3 / 2$ & 0 & $1 / 6$ & 0 & $-1 / 6$ & $17 / 6$ \\
\hline$x_{2}$ & 0 & 1 & $-1 / 4$ & 0 & $-1 / 4$ & 0 & $-1 / 4$ & $5 / 4$ \\
\hline$x_{4}$ & 0 & 0 & 2 & 1 & $1 / 3$ & 0 & $-1 / 3$ & $20 / 3$ \\
\hline$s_{2}$ & 0 & 0 & $3 / 4$ & 0 & $-1 / 4$ & 1 & $-1 / 4$ & $1 / 4$ \\
\hline$z_{j}-c_{j}$ & 0 & 0 & $11 / 2$ & 0 & $25 / 6$ & 0 & $5 / 6$ & $47 / 6$ \\
\hline
\end{tabular}

The CE from Table 7 will be given by:

$33 x_{3}+25 s_{1}+5 s_{4}=5+6 i, i=0,1, \ldots, 7$

One can easily verify that the optimal solution is again at the same point as was earlier for $i=$ 5 , we have $x_{1}=4, x_{2}=3, x_{4}=9, s_{2}=2, s_{4}=7, x_{3}=s_{1}=0, z(\operatorname{Mod})=4$ but the integer point is the same.

\subsection{Example 3}

Consider a bi-objective model.

$$
\operatorname{Max} z=\left\{z_{1}, z_{2}\right\} \text { where } z_{1}=3 x_{1}+2 x_{2} \text {, and } z_{2}=x_{1}+3 x_{2}
$$

Subject to: $2 x_{1}+3 x_{2} \leq 11, x_{1} \leq 4, x_{2} \leq 3, x_{1}, x_{2} \geq 0$ and integer.

Note the above problem has been analysed as Example 1 where the objective $\mathrm{z}$ was the same as $z_{1}$ in (24). Here we will illustrate that we need not reconsider the full convex space as it has already been investigated with respect to the objective $z_{1}$ and now only region 3 will be scanned for integer points. Region 3 convex space will be a sub-space of defined by and shown in Figure 1:

$$
2 x_{1}+3 x_{2} \leq 11, \quad x_{1} \leq 4, x_{2} \leq 3,3 x_{1}+2 x_{2} \leq 8, x_{1}, x_{2} \geq 0 \text { and integer }
$$

The final simplex result will be given as in Table 8 .

Table 8. Optimal solution with respect to $z_{2}$.

\begin{tabular}{|c|c|c|c|c|c|c|c|}
\hline & $x_{1}$ & $x_{2}$ & $s_{1}$ & $S_{2}$ & $S_{3}$ & $S_{4}$ & RHS \\
\hline$s_{1}$ & 0 & 0 & 1 & 0 & $-5 / 3$ & $-2 / 3$ & $2 / 3$ \\
\hline$s_{2}$ & 0 & 0 & 0 & 1 & $2 / 3$ & $-1 / 3$ & $10 / 3$ \\
\hline$x_{2}$ & 0 & 1 & 0 & 0 & 1 & 0 & 3 \\
\hline$x_{1}$ & 1 & 0 & 0 & 0 & $-2 / 3$ & $1 / 3$ & $2 / 3$ \\
\hline$z_{j}-c_{j}$ & 0 & 0 & 0 & 0 & $7 / 3$ & $1 / 3$ & $29 / 3$ \\
\hline
\end{tabular}

The CE will be given by: $7 s_{3}+s_{4}=2+3 i$. 
International Journal of Mathematical, Engineering and Management Sciences

Vol. 6, No. 2, 611-620, 2021

https://doi.org/10.33889/IJMEMS.2021.6.2.037

Here we reconsider the subspace as shown in Figure 1. We introduce one more objective $z_{2}=x_{1}+$ $3 x_{2}$ subject to the same constraints as in Example 1. Since we have explored part of the convex space with respect to the objective $z_{1}$, we need not scan fully the convex space but will consider only the Region 3 as was explained earlier. Region 3 is formed by the points $(0,0),(8 / 3,0)$ and $(0,4)$, which is a fractional region to be searched.

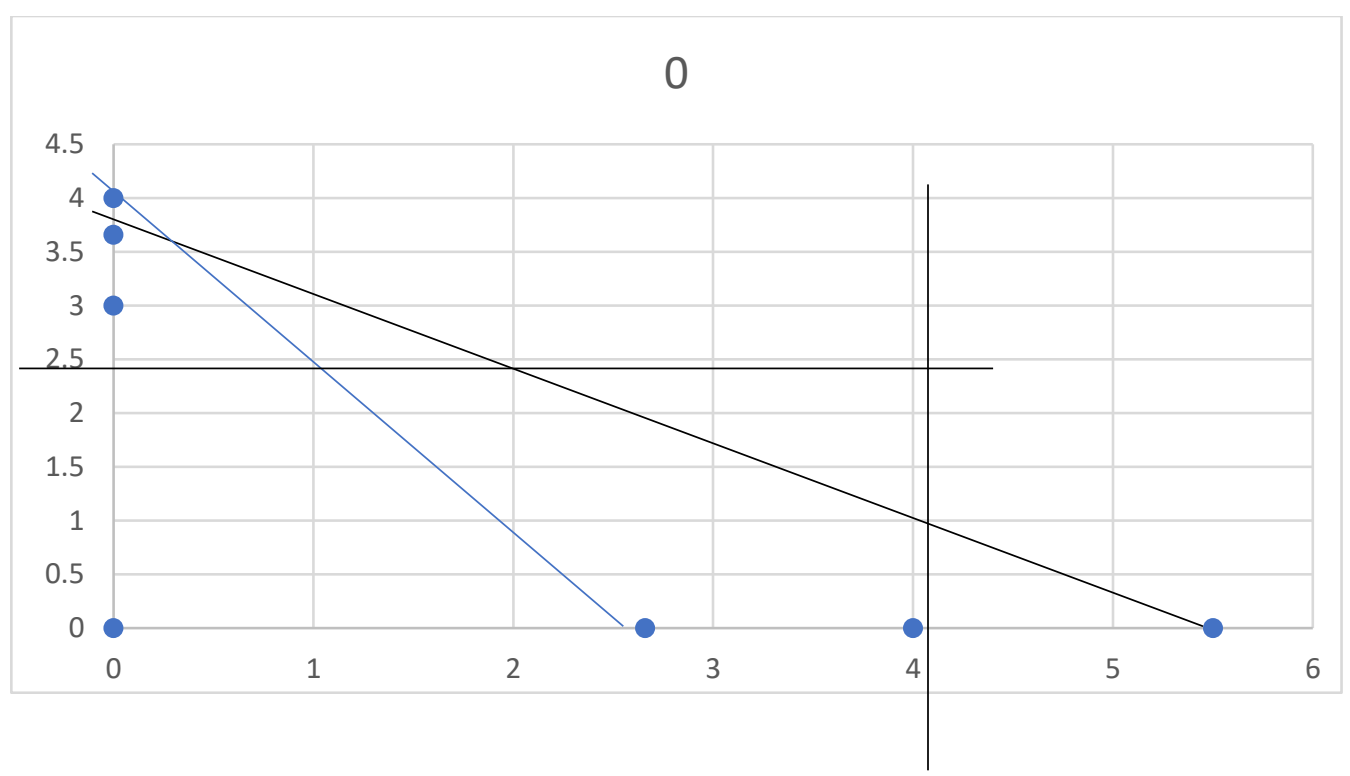

Figure 1. Reduced region to be explored.

\section{Conclusion}

In this paper, a few more properties associated with a characteristic equation in context of single and multi-objective linear integer program have been identified and numerically illustrated. We also identified why the CE approach is not applicable to special LPs like an assignment and transportation models. Ranked optimal solutions have been discussed in Kumar et al. (2018, 2020), Murty (1968) and some ideas on information recycle discussed by Kumar $(2005,2006)$ may be of value for extending existing optimization approaches to find ranked-optimal solutions. These ideas will be attempted in a subsequent publication.

\section{Conflict of Interest}

The authors declare that there is no conflict of interest for this publication.

\section{Acknowledgements}

The authors are grateful to the referees for their constructive suggestions and helpful comments. 
International Journal of Mathematical, Engineering and Management Sciences

Vol. 6, No. 2, 611-620, 2021

https://doi.org/10.33889/IJMEMS.2021.6.2.037

\section{References}

Al-Hasani, A., Al-Rabeeah, M., Kumar, S., \& Eberhard, A. (2020). Rank-based solution methods and their applications in determination of non-dominated points set for a multi-objective integer programming model. International Journal of Mathematical, Engineering and Management Sciences, 5(6), 12491269.

Al-Rabeeah, M., Kumar, S., Al-Hasani, A., Munapo, E., \& Eberhard, A. (2019). Bi-objective integer programming analysis based on the characteristic equation. International Journal of System Assurance Engineering and Management, 10(5), 937-944.

Gomory, R.E. (1958). Outline of an algorithm for integer solutions to linear programs. Bulletin of the American Mathematical Society, 64(5), 275-278.

Kumar, S. (2005). Information recycling mathematical methods for protean systems: a path-way approach. South African Journal of Industrial Engineering, 16(2), 81-101.

Kumar, S. (2006). Information recycling mathematical methods for protean systems: a path-way approach to a geometric program. South African Journal of Industrial Engineering, 17(2), 127-143.

Kumar, S., \& Munapo, E. (2012). Some lateral ideas and their applications for developing new solution procedures for a pure integer programming model, In Proceedings of Herbal International Conference on Applications of Mathematics and Statistics for Intelligent Solutions through Mathematics and Statistics, Excel India Publishers, pp. 13-21.

Kumar, S., Al-Hasani, A., Al-Rabeeah, M., \& Eberhard, A. (2020, March). A random search method for finding ' $\mathrm{K} \geq 2$ 'number of ranked optimal solution to an assignment problem. In Journal of Physics: Conference Series, 1490(1), 1-13. doi:10.1088/1742-6596/1490/1/012063.

Kumar, S., Munapo, E., \& Jones, B.C. (2007). An integer equation controlled descending path to a protean pure integer program. Indian Journal of Mathematics, 49(2), 211-237.

Kumar, S., Munapo, E., \& Jones, B.C. (2009). Descending hyper-plane approach for solving a binary integer program. International Journal of Mathematical Modeling, Simulation and Applications, 2(1), 53-62.

Kumar, S., Munapo, E., Lesaoana, M., \& Nyamugure, P. (2018). Some innovations in OR methodology: linear optimization. Lambert Academic Publishing, Beau Bassin.

Murty, K.G. (1968). An algorithm for ranking all the assignments in order of increasing cost. Operations Research, 16(3), 682-687.

Nyamugure, P., Munapo, E., Lesaoana, M., \& Kumar, S. (2017). A heuristic for a mixed integer program using the characteristic equation approach. International Journal of Mathematical, Engineering and Management Sciences, 2(1), 1-16.

Schrijiver, A. (1986). Theory of linear and integer programming. John Wiley, New York.

Shogan, A.W. (1988). Management science. Prentice Hall, New Jersey. 